\title{
Depinning and creeplike motion of wetting fronts in weakly vibrated granular media
}

\author{
Alexander S. Balankin, ${ }^{1, *}$ E. García Otamendi, ${ }^{1}$ D. Samayoa, ${ }^{1}$ J. Patiño, ${ }^{1}$ and M. A. Rodríguez ${ }^{2}$ \\ ${ }^{1}$ Grupo “Mecánica Fractal," Instituto Politécnico Nacional, México D.F., México 07738 \\ ${ }^{2}$ Instituto de Física de Cantabria (IFCA), CSIC-UC, E-39005 Santander, Spain
}

(Received 10 October 2011; published 23 March 2012)

\begin{abstract}
We study the effect of weak vibrations on the imbibition of water in granular media. In our experiments, we have observed that as soon as the vibration is applied, an initially pinned wetting front advances in the direction of imbibition. We found that the front motion is governed by the avalanches of localized intermittent advances directed at $45^{\circ}$ to the imbibition direction. When the rescaled gravitational acceleration of vertical vibrations is in the range of $0.81 \leqslant G \leqslant 0.95$, we observed an almost steady motion of wetting front with a constant velocity $v_{c r}(G) \propto \exp (-1 / G)$ during more than 20 min, whereas at lower accelerations $(0.5 \leqslant G \leqslant 0.8)$ the front velocity decreases in time as $v \propto t^{-\delta}$. We suggest that the steady motion of an imbibition front in a weakly vibrated granular medium can be treated as a creep motion associated with nonthermal temporal fluctuations of packing density in a weakly vibrated granular medium.
\end{abstract}

DOI: 10.1103/PhysRevE.85.036313

PACS number(s): 47.56.+r, 45.70.-n, 68.08.Bc, 05.70.Ln

\section{INTRODUCTION}

Fluid imbibition in porous media has tremendous importance in fields as diverse as the petroleum and construction industries, the paper and textile technologies, and soil science and hydrology, among others (for review, see [1], and references therein). At the same time, the imbibition phenomena bring forward challenging questions from a fundamental point of view, in particular in statistical physics of nonequilibrium systems (see Refs. [2-16]). Accordingly, for more than 100 years, extensive experimental and theoretical studies have been performed to understand the mechanisms of imbibition. Nonetheless, some aspects of the imbibition processes remain unknown. This stimulates continued research efforts aimed at better understanding the imbibition and related phenomena (see, for example, Refs. [4-14,17-22] and references therein).

Imbibition is a process by which a wetting fluid is drawn into a capillary or porous medium due to capillary action $[1,11,23]$. The spontaneous imbibition flow is driven solely by the potential generated by the wettability difference at the solid wall with respect to a lesser wetting fluid (or gas) occupying the space. The forced imbibition is governed by a combination of capillary phenomena and an externally enforced flow rate or pressure difference. The physics of imbibition is governed by the ratio of gravity and capillary forces, known as the Bond number Bo $=g \Delta \rho d^{2} / \sigma$, where $g$ is the gravity constant, $d$ is the pore radius, $\Delta \rho$ is the difference of fluid densities, and $\sigma$ is the surface tension associated with the interface between the wetting and nonwetting fluids [5]. Imbibition processes are also strongly dependent on the capillary number $\mathrm{Ca}$ defined as the ratio of viscous and capillary forces $\mathrm{Ca}=\mu V / \sigma$ with $\mu$ being the fluid dynamic viscosity and $V$ the fluid velocity $[5,18,24]$. Furthermore, if the viscous forces play a role in both fluids, a consideration of the viscosity ratio $M=\mu_{w} / \mu_{n w}$ is also necessary, because the viscous forces can either stabilize or destabilize the fluid-fluid interface [24]. Depending on the flow conditions and pore radius, extremely different flow regimes occur [5]. When $M \gg 1$, Bo $\ll 1$, and $\mathrm{Ca} \ll 1$, the

*abalankin@ipn.mx capillary forces are dominant and the spontaneous imbibition occurs in the Washburn regime in which the mean position (height) of the wetting front with respect to the free fluid level increases in time as

$$
H^{2}(t)-H_{0}^{2}=\frac{\kappa P_{c}}{\mu}\left(t-t_{0}\right),
$$

for times $t_{0} \leqslant t<\tau_{g}=P_{c} \mu /(g \rho)^{3} \kappa$, where $P_{c}=2 \sigma \cos \theta / d$ is the capillarity pressure, $\theta$ is the contact angle, $\kappa$ is the intrinsic permeability of the porous medium, and $H_{0}$ is the wetting front position at $t=t_{0}$. Equation (1) follows from the balance of the viscous drag force and the Laplace pressure in the Darcian flow (see Refs. [1,3]). In the absence of evaporation, the equilibrium height,

$$
H_{g}=\frac{P_{c}}{g \rho},
$$

is approached exponentially as

$$
H(t) \propto H_{g}\left[1-\exp \left(-\frac{t}{\tau_{g}}\right)\right],
$$

for times $t>\tau_{g}$, where $\tau_{g}=\mu H_{g} / \kappa g \rho^{2}$ [3]. Fluid evaporation can cause the pinning of the wetting front at the height $H_{g e}$ less than $H_{g}$ [25]. Moreover, evaporation leads to a breakdown of the liquid conservation law [7]. Nonetheless, in this case, the Washburn-like regime $H \propto t^{1 / 2}$ is also observed for $H \ll$ $H_{g e}$, while the pinning height is approached exponentially [3]. Although both the gravity and the evaporation pin the interface at a given height, the physics behind these two effects is quite distinct (see Refs. [3,7]). Consequently, the time and length scales coming from gravity and evaporation can be very different [7].

The presence of quenched disorder in a porous medium gives rise to random capillary forces that roughen the moving wetting front [26]. Structural heterogeneities control the global flow process at different scales (see Refs. [7,18]). Specifically, the capillary disorder acts only at the imbibition front, whereas the permeability disorder controls the flux of liquid from the reservoir to the front [18]. Furthermore, the pinning height in a disordered porous medium $\left(H_{p}\right)$ is expected to be lower than 
the height $H_{g e}$ predicted by the mean field analysis accounting for the effects of gravity and evaporation only (see Ref. [25]). Kinetic roughening and pinning are fundamental problems of nonequilibrium statistical physics. In this context, the studies of wetting fronts in porous media produce essential results for the understanding of some nonequilibrium phenomena (see Refs. [1,26,27]).

The dynamics of a wetting front in a porous medium can be understood as a sequence of avalanches [1,13-18]. In fact, at any given time, only a small localized portion of the interface is moving quickly. Propagation by avalanches in imbibition and their size distribution with one fixed scale has been found in experiments and discussed in Refs. [14-18,28,29]. Avalanche dynamics introduces long-range correlations in the moving front roughness. Consequently, it was found that rough fronts commonly display a statistical scale invariance over a wide but bounded range of length scale [26-30], while the kinetic roughening obeys dynamic scaling ansatz characterized by a set of critical exponents [26,30]. Furthermore, when a driving force $F$ is close to the pinning strength $F_{P}$, the rough interface is expected to move with velocity

$$
v \propto\left(F-F_{P}\right)^{\beta},
$$

where $\beta>0$ is the critical scaling exponent [30]. Notice that when the pinning is controlled by gravitational force $F_{P}=$ $\rho g H(t)$, relation (3) can be derived from Eq. (4) with $\beta=1$ [2]. Nonetheless, the authors of Ref. [2] suggested that Eq. (4) with anomalously larger exponent $\beta=7.1 \pm 1.2$ provides a better fit to the experimental data than the exponential function (3).

The interface becomes pinned when the driving force is less than the pinning strength $\left(F_{P}>F\right)$. However, even in this case, the interface can move due to the temporal fluctuations of pinning and/or driving forces [28]. Depinning and creep motion of rough interfaces near the pinning threshold were observed in many systems of different nature (see, for review, Refs. [31,32]). These include the magnetic domain wall motion in thin films [33], the driven vortex lattices and charge density waves [34], the dynamics of dislocation in an inhomogeneous environment [35], and many others. In all these systems the competition between the spatial disorder and the interface elasticity leads to pinned configurations characterized by a roughness exponent $\zeta_{P}$, which depends only on the universality class of the disorder, the dimension of the interface, and the nature of the elastic forces [36]. However, the pinning barriers can be overcome due to temporal fluctuations, resulting in a moving interface even below the critical driving field.

The temporal fluctuations of pinning strength are commonly associated with thermal fluctuations in the system (see Refs. [33-36]). Due to thermal fluctuations, the creep velocity obeys the Arrhenius behavior $v_{c r} \propto \exp [-U(F) / T]$ with energy barrier $U\left(F<F_{C}\right)>0$ dependent on the driving field [36]. At the same time, there are many complex systems where thermal fluctuations are small enough that the temperature of the external bath can be considered zero [37-39]. Nevertheless, in this case one can also expect to observe creep phenomena, but associated with temporal fluctuations of a nonthermal nature, e.g., quantum collective creep [37] and radiationinduced nonthermal creep in a high radiation environment [38].
In a porous granular medium, the temporal fluctuations can be induced by weak vibrations [39-43]. The effects of acoustic vibrations on the fluid flow through porous media have been studied in the past few decades (see Refs. [44-48] and references therein). Most of these studies were focused on the use of acoustic waves as a technique for enhanced oil recovery. Specifically, oil recovery from highly fractured carbonate reservoirs with low-permeability matrix blocks is an example where the spontaneous imbibition of water is an important improved oil recovery technique [49]. It was found that irradiation of ultrasonic energy leads to faster motion of oil in porous media at laboratory as well as reservoir scales $[45,48]$. In this context, it was suggested that the energy of vibrations can change the properties of the media, as well as the liquid-liquid, liquid-solid, and gas-liquid interactions [45]. However, the mechanisms of these changes are not yet well understood (see [48] and references therein).

In this paper we report the results of an experimental study of wetting fronts in weakly vibrated granular media. The study is focused on the effect of weak vibrations on the wetting front depinning and motion after the vibrations are applied. The paper is organized as follows. In Sec. II, we describe the details of experiments performed in this work. Experimental findings are reported in Sec. III. Section IV is devoted to the discussion of experimental results. A brief summary of the main findings and some conclusions are given in Sec. V.

\section{EXPERIMENTAL DETAILS}

Our basic research goal was to observe and study the depinning and motion of initially pinned water imbibition fronts in granular media after weak vibrations are applied. The experimental setup used for this purpose is shown in Fig. 1(a). It consists of a rectangular glass cell filled with sand and fixed to the frame rigidly mounted on the platform. To avoid the escape of sand grains, the bottom of the cell is closed by a textile filter. The cell bottom is submerged into a water reservoir in which the level of water is kept
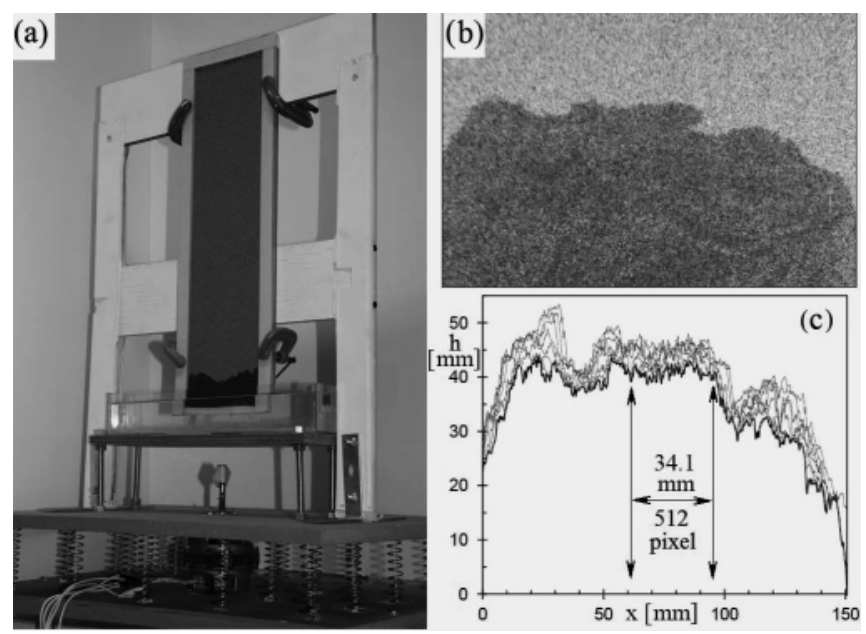

FIG. 1. (a) The experimental setup. (b) The snapshot of pinned wetting front. (c) The configurations of wetting front moving in weakly vibrated granular medium $(G=0.84)$; the thick curve represents the pinned front. 
constant during an experiment. All height measurements are made taking into account this reference level. The cell can be subjected to vertical vibration by an electric motor. The cell acceleration is measured by two accelerometers placed on the frame. Accordingly, the shaking intensity is characterized by the maximum vertical acceleration normalized by gravity $G=A \omega^{2} / g$, where $A$ and $\omega$ are the vibration amplitude and frequency, respectively.

Specifically, we used rectangular cells of height $H_{\text {cell }}=$ $50 \mathrm{~cm}$ and width $W_{\text {cell }}=15 \mathrm{~cm}$, while the cell aperture (distance between glass plates, $d_{\text {cell }}$ ) was varied in the range from 0.8 to $3 \mathrm{~mm}$. In each experiment, the aperture was set by using calibrated nylon fibers at the lateral long sides and sealed with epoxy resin, in order to get a narrow slit, with inlet and outlet in the shorter sides. Before each experiment, the glass plates were rinsed with water, and the remaining water film was displaced from the surface with a nitrogen jet. The cell inside was filled up with sea sand (one grain density is of approximately $\rho_{s}=3.26 \mathrm{~g} / \mathrm{cm}^{3}$ ) with grains of different sizes in the range of $0.03 \leqslant a \leqslant 0.4 \mathrm{~mm}$. The gain size distribution appears to be an almost log-normal distribution with a mean about of $0.15 \mathrm{~mm}$. To avoid the effect of capillary bridges among the sand grains (see [50]), the sand was dried in ambient conditions (air temperature of $23 \pm 5^{\circ} \mathrm{C}$ and relative humidity of air less than $60 \%$ ) for a few days and all experiments were carried out under ambient temperature of $23 \pm 5^{\circ} \mathrm{C}$, atmosphere pressure, and relative humidity of air of $53 \pm 8 \%$. We use a new portion of sea sand for each experiment. The cells filled with sand were weighted and the mean packing density was calculated as the volume fraction of solid sand in the cell $v_{s}=M / \rho_{s} V_{\text {cell }}$, where $V_{\text {cell }}=H_{\text {cell }} W_{\text {cell }} d_{\text {cell }}$ is the cell volume and $M$ is the mass of the sand in the cell. Maximal packing density of polydisperse grains is determined by the grain geometry and size distributions [51]. In practice, however, it is difficult to achieve the maximum packing density in cells with apertures of the order of the maximum size of grains. Accordingly, in this work, the mean porosity of sand packing $\phi=1-v_{s}$ was varied in the range from 30 to $60 \%$, while vibrations were applied to achieve the closest packings with $\phi \leqslant 50 \%$. Furthermore, we noted that the packing density is not homogeneous, but shows visible spatial variations with the characteristic scale of $40 \pm 10 \mathrm{~mm}$.

Once the cell bottom is submerged to a depth of about $10 \mathrm{~mm}$ below the water level in the reservoir, the water impregnates the sand in the forced imbibition regime until the level of the wetting front coincides with the water level in the reservoir at time $t_{0}$. Thereupon, the wetting front motion is governed by spontaneous imbibition. The positions and configurations of the moving wetting front $z(x, t)$ were determined from the digital photographs [see Fig. 1(b)] taken every $30 \mathrm{~s}$ by a digital camera with a resolution of 15 pixels $/ \mathrm{mm}$. All snapshots were digitized and plotted as univalued functions $h(x, t)=[\max z(x, t)-\min z(x, t=0)]$ [see Fig. 1(c)] [52].

We allow the wetting front to rise until it stops. It should be pointed out that, in our experiments, the stopping can be attributed to the effect of gravitation and the pinning on heterogeneities in granular media, rather than to the effects of water evaporation. In fact, in contrast to the imbibition in a paper sheet, when the net loss of fluid mass per unit time due to evaporation increases as the imbibition front is advanced (see Refs. $[3,53]$ ), in our experiments the water can evaporate from the gap on the cell top only. The area of this gap $W_{\text {cell }} d_{\text {cell }}$ is rather small $\left(d_{\text {cell }} \ll H_{\text {cell }}\right)$, such that the evaporation force is expected to be negligible in comparison to the gravitational one.

After the wetting front was completely pinned and no change in either height or shape of the interface between the wet and dry regions was observed during $10 \mathrm{~min}$, the cell was quasiharmonically vibrated in the vertical direction at a frequency of $\omega=860 \pm 90 \mathrm{~Hz}$ at amplitudes providing the rescaled gravitational acceleration $G=A \omega^{2} / g$ (measured with an accuracy of $10^{-2} \mathrm{~g}$ by a VB-8201HA accelerometer) in the range of $0.5 \leqslant G<1$. We noted that the amplitude of water waves in the reservoir was less than $2 \mathrm{~mm}$ in all experiments.

As soon as the vibration is applied, the initially pinned wetting font advances in the vertical direction. The motion of the wetting front in the vibrated granular medium was monitored during at least $25 \mathrm{~min}$ [54]. To avoid the effect of cell edges, only the central third part of the moving front was analyzed. Furthermore, to avoid the effect of large scale heterogeneities in the sand packing, the scaling properties of wetting fronts were measured using the front fragments,

$$
\widehat{h}(x, t)=\max _{x_{0} \leqslant x \leqslant x_{0}+512} z(x, t)-\min _{x_{0} \leqslant x \leqslant x_{0}+512} z(x, t=0),
$$

of the length $L=512$ pixels $=34.1 \mathrm{~mm}$ with the minimal inclination [see Figs. 1(c) and 2]. Although the coordinate $x_{0}$ is different in different experiments, for simplicity, we always put $x_{0}=0$ (see Fig. 2). The roughness exponents of pinned $\left(\zeta_{P}\right)$ and moving $\left(\zeta_{c r}\right)$ wet fronts were determined with the help of the commercial software BENOIT1.3 [55] by the variogram $V \propto \Delta^{2 \zeta}$, roughness length $\sigma \propto \Delta^{\zeta}$ methods where $V=\left\langle[h(x)-h(x+\Delta)]^{2}\right\rangle$ is the average value of the squared difference between pair points at distance $\Delta ; \sigma(\Delta)$ is the standard deviation measured in the window of size $\Delta$ (root-mean square roughness) and the wavelet transform method $(\zeta$ is equal to the slope of decay for discrete wavelet coefficients belonging to the wavelet best basis [56]). It is pertinent to note that the difference in the values of the roughness exponent obtained by three different methods was always within the error range of data reported in Sec. III.

It should be pointed out that although the experimental data reported in Sec. III were obtained in cells with apertures of $1 \mathrm{~mm}$ filled by sand with grain sizes in the range of $0.09 \leqslant$ $d \leqslant 0.4 \mathrm{~mm}$ (with the mean of $0.15 \mathrm{~mm}$ ) and the packing porosity about $45 \pm 5 \%$, qualitatively the same phenomena were observed in the preliminary experiments with cells with apertures of $0.8 \leqslant d_{\text {cell }} \leqslant 3 \mathrm{~mm}$ which were filled with the sand up to different packing porosities (from 30 to $60 \%$ ).

\section{EXPERIMENTAL FINDINGS}

We found that at the stage without vibrations, a wetting front advances due to spontaneous imbibition in an almostWashburn regime [Eq. (1)]. In all experiments reported below, the wetting fronts were completely pinned after roughly $1 \mathrm{~h}$. The maximum elevation of the pinned front with respect to the water level in the reservoir $H_{M}=\max _{0 \leqslant x \leqslant 2500} z(x)$ was 

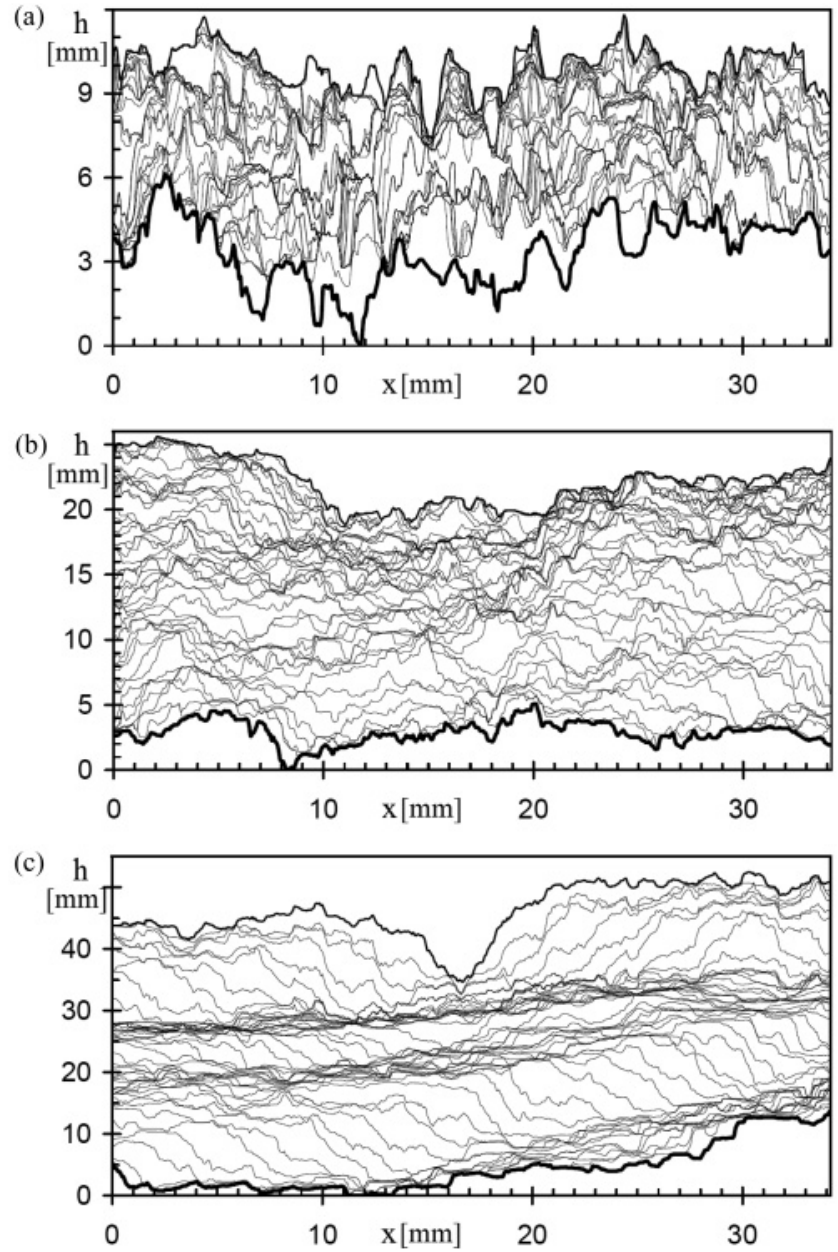

FIG. 2. Time evolution of analyzed fragments [Eq. (5)] of the wetting fronts in granular media vibrating with the adimensional acceleration $G=$ (a) 0.84 , (b) 0.88 , and (c) 0.91 .

always of approximately $20 \pm 5 \mathrm{~cm}$. Notice that for a medium with a pore size of $0.15 \mathrm{~mm}$ the equilibrium height defined by Eq. (2) is expected to be of the order of $20 \mathrm{~cm}$. Hence, gravitation plays a dominant role in the pinning of the wetting front, while the disorder in porous media determines the front roughness.

We noted that the global width of pinned fronts $W_{P}=$ $\max _{0 \leqslant x \leqslant 2500} z(x)-\min _{0 \leqslant x \leqslant 2500} z(x)$ was of the order of $50 \mathrm{~mm}$ [see Fig. 1(c)], whereas the global width $\widehat{W}_{P}$ of analyzed fragments [Eq. (5)] never exceeded $5 \mathrm{~mm}$ [see Figs. 1(c) and 2]. The large values of $W_{P}$ can be attributed to the effects of edges and the large scale heterogeneities in the sand packing. Although, generally, the imbibition front roughness is characterized by two independent scaling exponents (see Refs. $[7,8,13,16,57])$, only the local roughness exponent was measured in this work. Specifically, we found that the local roughness of the pinned wetting fronts is characterized by the local roughness exponent $\zeta_{P}=0.50 \pm 0.06$ (see Fig. 3). Notice that this value coincides with the prediction of the directed percolation depinning model, as well as with the results of early imbibition experiments in two plus one dimensions (see Refs. $[28,58]$ ), rather than with the experimental results and theoretical prediction $\zeta_{P}=0.63$ and for imbibition in one
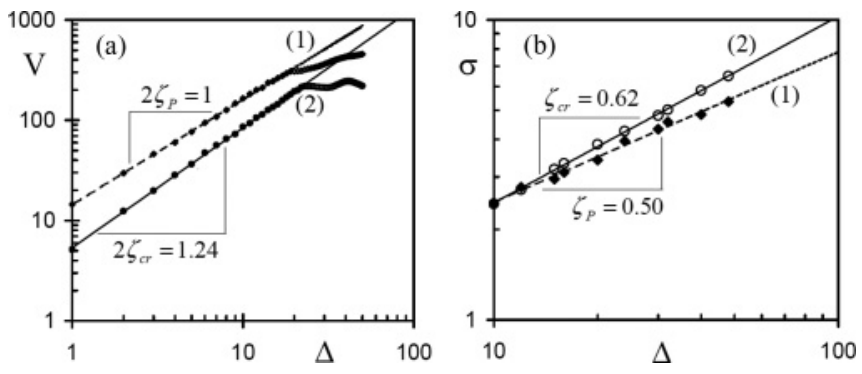

FIG. 3. Log-log plots of (a) variogram $V$ (in arbitrary units) and (b) root-mean square roughness $\sigma$ (in arbitrary units) versus $\Delta$ (in pixels) for the pinned wet front [curve (1)] and wet fronts moving with constant velocity in weakly vibrated $(G=0.87)$ granular medium [curve (2)].

plus one dimensions (see Refs. [25,28,30,58]). Therefore, our finding indicates that the granular medium used in this work is essentially three dimensional; nonetheless the ratio of the cell gap thickness to the mean grain size is relatively small $\left(d_{\text {cell }} / a_{\text {mean }}=6.7\right)$. In fact, however, the imbibition flow differs from a pure three-dimensional flow, at least in some aspects, due to the edge effects in the lateral direction, which cannot be avoided in our experiments.

As soon as the vibration is applied, the initially pinned wetting front advances in the vertical direction. Figure 2 shows the evolution of wetting fronts in the vibrated granular medium. We found that when the gravitational acceleration is in the range of $0.5 \leqslant G<0.8$, the wetting front velocity in
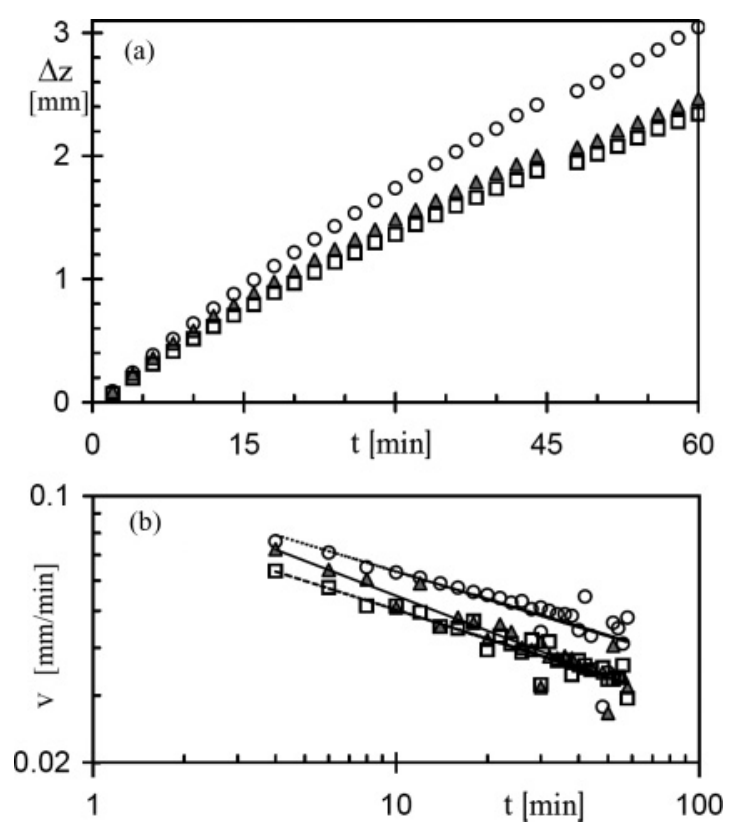

FIG. 4. (a) The graphs of wetting front position $\Delta z=$ $\langle\overline{z(x, t)}-\overline{z(x, 0)}\rangle$ versus time $t$ and (b) $\log$-log plots of wetting front velocity [Eq. (6)] versus time in granular media vibrating with acceleration of $G=0.5$ (squares), 0.7 (triangles), and 0.78 (circles). Experimental data are averaged over three experiments with each $G$; straight lines represent the data fitting as $v \propto t^{-\delta}$ with the scaling exponent $\delta=0.25$ (dashed), 0.3 (solid), and 0.24 (dotted), respectively. 


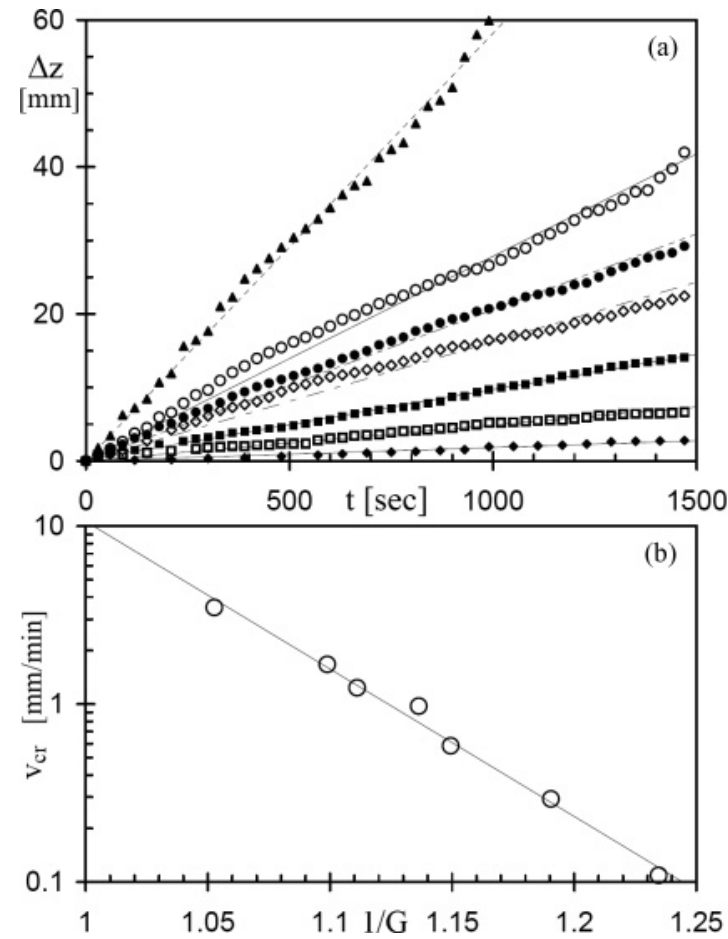

FIG. 5. (a) The graphs of wetting front position versus time $t$ for seven values of gravitational acceleration (from bottom to top $G=0.81,0.84,0.87,0.88,0.9,0.91$, and 0.95 ); experimental data are averaged over three experiments with each $G$; straight lines represent linear fittings $\Delta z=v_{c r} t$ with $v_{c r}=0.109$, $0.294,0.583,0.974,1.236,1.672$, and $3.493 \mathrm{~mm} / \mathrm{min}$, respectively. (b) Semilog plot of creep velocity $v_{c r}$ versus the inverse of adimensional acceleration $(1 / G)$; the fitting line indicates exponential decay $v_{c r}=2 \times 10^{9} \exp (-19.1 / G)$.

the vertical direction, defined as

$$
v=\overline{\langle\bar{h}(x, t+\tau)}-\overline{\widehat{h}(x, t)}\rangle / \tau,
$$

decreases in time as $v \propto t^{-\delta}$ with the scaling exponent $\delta=$ $0.27 \pm 0.04$ (see Fig. 4); here the overbar denotes the average over $x$ for the profile of size $L=512$ pixels and brackets denote the average over a set of experiments with the same $G$. We also noted that the global width of moving front fragment [Eq. (5)], defined as

$$
\widehat{W}(L=512, t)=\max _{0 \leqslant x \leqslant 512} \hat{h}(x, t)-\min _{0 \leqslant x \leqslant 512} \hat{h}(x, t),
$$

only slightly fluctuates in time (see Fig. 2). The local roughness exponent of moving wetting front also varies with time in the range of $0.5 \leqslant \zeta_{v}(t) \leqslant 0.6$, but no systematic dependence of $\zeta_{v}(t)$ on time or $G$ was observed.

In contrast to this, when the gravitational acceleration is in the range of $0.81 \leqslant G \leqslant 0.95$, the wetting front moves with an almost constant velocity $\left(v=v_{c r}(G)=\right.$ const) controlled by the rescaled gravitational acceleration $G$ (see Fig. 5). We found that the roughness of wetting front moving in vibrated medium with a constant velocity is characterized by the local roughness exponent

$$
\zeta_{c r}=0.62 \pm 0.06>\zeta_{P}
$$

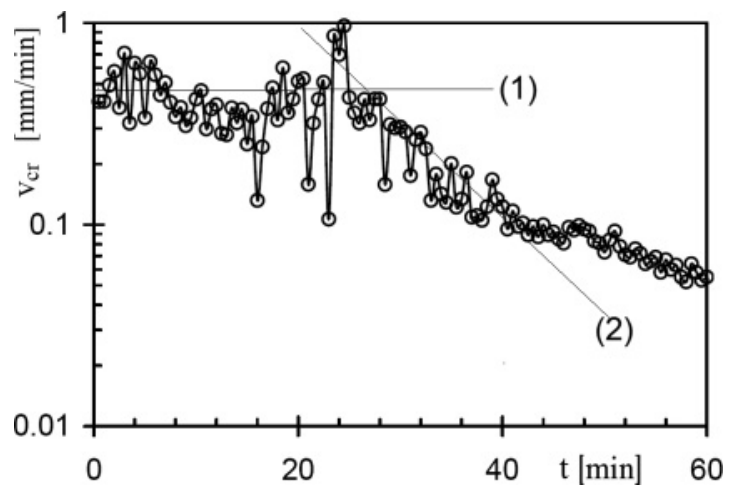

FIG. 6. Semilog graph of wetting front velocity [Eq. (6)] versus time in granular medium vibrating with $G=0.84$. Straight lines indicate the mean creep velocity during the first $24 \mathrm{~min}$ (1) and the decrease of mean velocity at larger times (2), respectively. One can see that creep velocity behavior is almost constant during $24 \mathrm{~min}$ and decreases after this time.

after a lapse of $5 \mathrm{~min}$ as the front is deppined (see Fig. 3). We also noted that in experiments with $0.81 \leqslant G \leqslant 0.87$, the wetting front velocity decreases after 24-30 minutes of motion (see Fig. 6), whereas in experiments with $G>0.87$, the front moves with constant velocity up to the top of the cell and the crossover from the constant to decreasing velocity was not observed. In this context, it is important to note that in all cases the crossover from the constant to decreasing velocity was observed when the height of the wetting front is rather higher than the expected equilibrium height of spontaneous imbibition [Eq. (2)]. Unfortunately, we were not able to determine the maximal height that the wetting front can achieve in a weakly vibrated medium [59].

\section{DISCUSSION}

The spontaneous imbibition in unconsolidated porous medium was widely studied experimentally and theoretically (see Refs. [60] and references therein). There are many factors affecting the water imbibition into the cell filled with sand (the cell gap thickness, the sand grain geometry and size distribution, the packing density, the boundary conditions, and the glass and sand wettability, among others). These factors can affect the effect of vibrations on the wetting front dynamics also. The wetting front motion in a vibrated medium can be also dependent on various parameters of vibrations, such as vibration spectrum, frequency, and amplitude (see Refs. [44-48]). Hence, a comprehensive study of the effect of vibrations on the wetting front dynamics should include the independent variations of each of these parameters. This paper, however, is limited to the study of the creeplike motion of wetting fronts in a weakly vibrated granular medium.

As follows from the graphs in Fig. 7, the wetting front in a weakly vibrated granular medium moves due to avalanches of localized intermittent advances. When the gravitational acceleration is in the range of $0.81 \leqslant G \leqslant 0.95$, the avalanches induce a constant velocity in the vertical direction $v_{c r}(G)$ (see Fig. 5). Avalanches also introduce the correlations in the wetting front roughness dynamics, such that the roughness of creeping fronts is characterized by $\zeta_{c r}>0.5$ (see Fig. 3). 

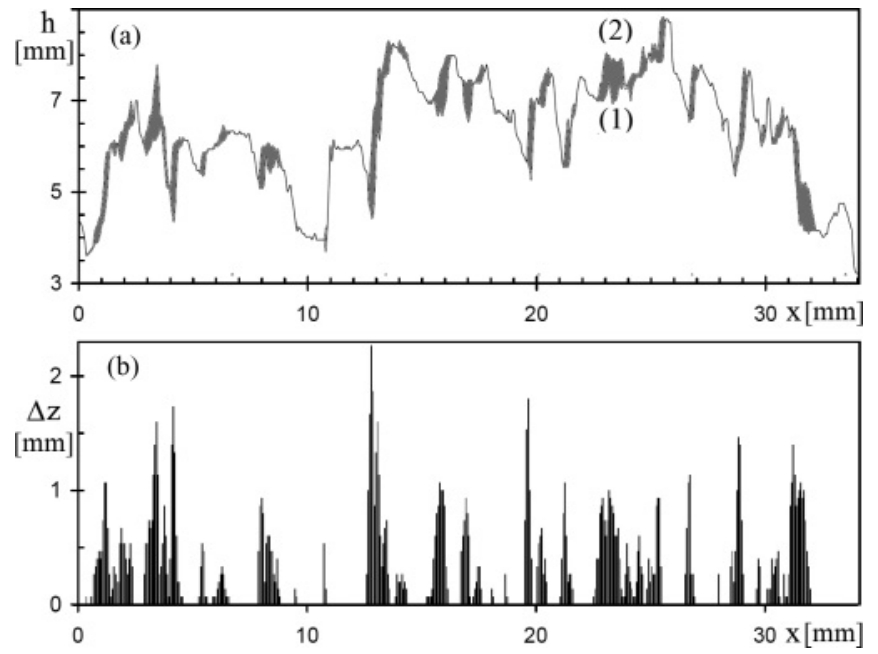

FIG. 7. Illustration of localized intermittent advances of the wetting front in a weakly vibrated $(G=0.84)$ granular medium: (a) wetting front configurations at $t=540$ [curve (1)] and $570 \mathrm{~s}$ [curve (2)], respectively; gray areas indicate the areas wetted due to the front advance during $\tau=30 \mathrm{~s}$ (the mean advance is $\overline{\Delta z(x, t=540, \tau=30)}=0.25 \mathrm{~mm})$; (b) spatial distribution of localized intermittent advances of wetting front $\Delta z(x, t, \tau=1)=$ $z(x, t+\tau=570)-z(x, t=540)$.

Furthermore, due to the existence of two characteristic length scales associated with the capillary and permeability disorders, the spatiotemporal distribution of avalanches is expected to obey exponentially decaying distribution functions of the form

$$
P\left(x_{i}\right) \propto x_{i}^{\chi_{i}} \exp \left(-x_{i} / x_{i C}\right),
$$

where $x_{i}\left(i=s, \ell, T_{A}\right)$ denotes the size $s$, the lateral extent $\ell$, and the duration $T_{A}$ of avalanches, respectively; the corresponding cutoffs $x_{i C}$ are expected to be functions of the flow velocity, while the scaling exponents $\chi_{i}$ are expected to be universal [14-16,28]. Unfortunately, the avalanche size distribution cannot be determined from the two-dimensional snapshots, because of the three-dimensional structure of avalanches in the two plus one dimensional granular medium. Nonetheless, we noted that the statistical distributions of areas of the two-dimensional projections of avalanches $\left(s_{P}\right)$ display the behavior [Eq. (9)], but with the value of the scaling exponent varying from experiment to experiment in the range of 0.9 to 2 [see Fig. 8(a)], while the expected value for imbibition in porous medium without vibrations is $\chi_{s}=1.5$ [28]). Moreover, we found that the avalanche duration distribution obeys the behavior [Eq. (9)] with the scaling exponent $\chi_{T}=4.5 \pm 0.3$ [see Fig. 8(b)]. This value is larger than the scaling exponent $\chi_{T}=2.18$ found in numerical simulations of avalanche dynamics in $2+1$ dimensions [28]. Furthermore, we found the avalanches are directed at $45^{\circ}$ to the vertical axis (see Figs. 2 and 9). This trend becomes more pronounced as $G$ increases. Consequently, we noted that the $45^{\circ}$ incline of avalanche tracks was observed in the numerical simulations of avalanche dynamics in fluid imbibition near the depinning transition (see [14]).

The possible mechanisms of vibration influence on the imbibition in a porous medium were discussed in Refs. [45-48].
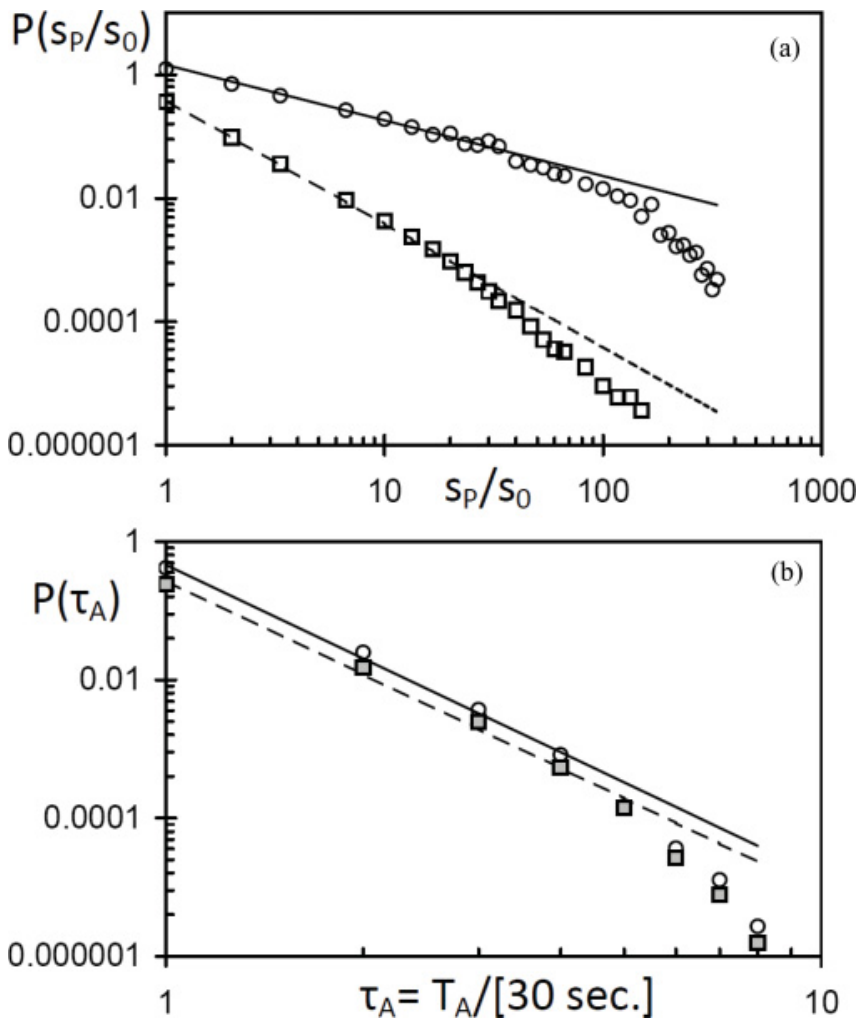

FIG. 8. Statistical distributions of (a) the areas of twodimensional projections of avalanches in granular medium vibrating with $G=0.87\left(s_{0}=0.018 \mathrm{~mm}^{2}\right.$; circles and squares correspond to two experiments realized under the same experimental conditions; straight lines with the slopes $\chi_{s_{P}}=0.9$ and 2.0 indicate the power-law trends of distributions for $s_{P} \ll s_{P C}$ ); (b) the avalanche durations in granular medium vibrating with $G=0.87$ (circles) and 0.91 (squares); experimental data are averaged over five experiments with each $G$. The slopes of straight lines are $\chi_{T}=4$.

Despite a great variety of factors associated with the effect of vibrations on the fluid dynamics, there are only two cardinally different scenarios of the wetting front depinning and acceleration. The first scenario is associated with the decrease of pinning strength $F_{P}$ and/or the increase of driving force $F$, such that the difference $\Delta F=F-F_{P}$ becomes positive and the front is expected to move with the time decreasing velocity

$$
v \propto(\Delta F)^{\beta} \propto t^{-\delta}
$$

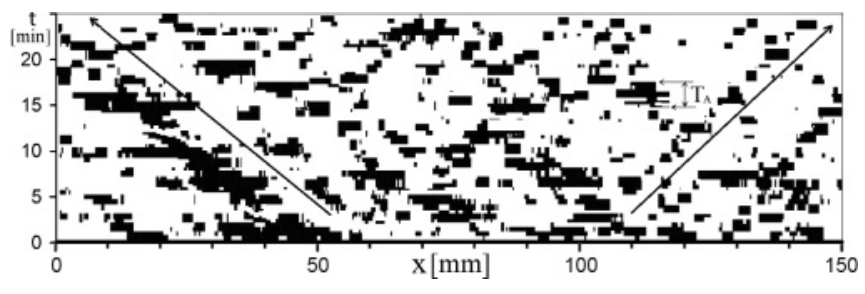

FIG. 9. Typical spatiotemporal distribution of the projections of water avalanches in a weakly vibrated granular medium (data correspond to experiment with $G=0.9$ ). Arrows indicate the avalanche directions, $\mathrm{T}_{\mathrm{A}}$ : avalanche duration. Compare this figure with 1(b) in Ref. [14] obtained by numerical simulations. 
where $\Delta F$ is a function of $G$ and $t$, while $\beta>0$ and $\delta>0$ are the characteristic scaling exponents [2]. In this work, the decelerating motion [Eq. (10)] of wetting fronts in vibrated granular media was observed when the gravitational acceleration is in the range of $0.5 \leqslant G<0.8$ (see Fig. 3).

The alternative scenario is associated with the pinning strength and/or driving force fluctuations related to the temporal fluctuations of medium density. In this case, one can guess that the wetting front will move in a creeping manner. The creep phenomenon, i.e., the steady-state response of complex systems with internal degrees of freedom to a small driving field, is one of the fascinating topics in statistical physics (see Refs. [31-38] and references therein). In fact, the pinning forces induce some effective energy barriers which suppress the interface motion. In the absence of temporal fluctuations in the system, the interface can move only if the driving force $F$ exceeds a threshold value $F_{P}$. However, the energetic barriers can be overcome due to temporal fluctuations, resulting in a moving interface even below the critical driving field. Consequently, the velocity of creep motion is expected to obey the Arrhenius-like behavior,

$$
v \propto \exp \left[-\frac{U(F)}{T_{\text {eff }}}\right],
$$

with the effective temperature $T_{\text {eff }}$ and a certain driving field dependent energy barrier $U\left(F<F_{C}\right)>0$ [31-38]. This barrier is related to the interface roughness exponent and to its origins. In recent times detailed studies of creep introduce classifications of these phenomena in universalities characterized by a given space-time scaling involving fractal structures with a rich dynamics (avalanches, etc.) [36]. Specifically, it has been shown that $U$ scales as

$$
U \propto\left(F / F_{C}\right)^{-\mu},
$$

where

$$
\mu=\frac{d-n+2 \zeta}{n-\zeta}
$$

is the creep exponent, $d$ is the topological dimension of the interface, $n$ is the $k$-space decay exponent of the elastic kernel (for long-range elasticity $n=1$, whereas for the shortranged case $n=2$ ), and $\zeta$ is the equilibrium roughness exponent which depends only on the universality class of the disorder, $n$ and $d$ [31,36]. In this context, we noted that the experimental value of the creeping wetting front roughness exponent [Eq. (8)] is consistent with the value $\zeta=(4-d) / 3=2 / 3$ expected for the equilibrium interface $(d=2)$ in a three-dimensional random environment [61].

We also noted that the motion of the wetting front with constant velocity in a weakly vibrated granular medium resembles the creep motion of interfaces moving through a quenched disordered medium. Although the wetting front motion with a constant velocity was observed for a rather small range of gravitational acceleration $(0.81 \leqslant G \leqslant 0.95)$, we found that the fast increase of front velocity as the vibration intensity increases is consistent with the exponential relation

$$
v_{c r} \propto \exp \left(-\frac{U_{0}}{G}\right),
$$

where $U_{0}$ is the fitting constant [see Fig. 5(b)]. Notice that relation (14) resembles the Arrhenius behavior [Eq. (11)] under the assumption that

$$
T_{\text {eff }} \propto G \text {. }
$$

It should be pointed out that the above assumption is consistent with the use of the dimensionless acceleration $G$ as the effective temperature of a weakly vibrated granular medium in the model of compaction dynamics under vertical tapping (see Refs. [62-64]) [65].

Generally, the effective temperature of a granular medium is associated with the medium density fluctuations and can be defined ether as the configurational temperature $T_{\text {conf }}=$ $(\partial S / \partial E)_{V}$, where $E$ is the energy, $V$ is the volume, and $S$ is the configurational entropy, or as the compactivity $X=$ $(\partial V / \partial S)_{E}$ conjugated of the volume in an analogous way as the temperature is the conjugate of the energy in usual thermal systems [66,67]. In a weakly vibrated granular medium the compactivity controls the volume fluctuations

$$
\left\langle\delta V^{2}\right\rangle=X^{2} \frac{\partial V}{\partial X},
$$

where $(\partial V / \partial X)$ plays the role of the specific heat in the equilibrium thermodynamics [39-41,66,67], while $X=0$ corresponds to the most compact situation, whereas $X=\infty$ is the less compact one. It should be emphasized that due to internal dissipation, the fluctuations defined by Eq. (16) can only be maintained by a constant supply of energy, e.g., by weak vibrations. The density of a granular medium under vibrations increases in time due to grain rearrangements [62-64] and so the compactivity is a decreasing function of time. Hence, the effective temperature $T_{\text {eff }}=X(G, V)$ of a weakly vibrated medium is expected to decrease in time. In view of this, it is somewhat surprising that the creep velocity is constant during the period of $t_{c r}(H, G)=25 \pm 5 \mathrm{~min}$ (see Fig. 6). However, the constant creep velocity may indicate that the decrease of $T_{\text {eff }}(t)$ is compensated by the time decrease of the ratio of the pinning to the driving forces. If so, from Eqs. (11)-(15) it follows that the ratio of the driving force to the pinning strength increases in time as

$$
\frac{F(t)}{F_{C}(t)}=\left(\frac{k G}{U_{0} T_{\mathrm{eff}}(t)}\right)^{1 / \mu},
$$

for times $t<t_{c r}(H, G)$, where $k$ is a constant. However, for longer times the increase of the driven force does not compensate for the decrease of effective temperature and the increase of gravitational force as the height of the wetting front increases. Accordingly, the creep velocity decreases in time for $t>t_{c r}(H, G)=25 \pm 5 \mathrm{~min}$. In this context, the increase of driven force $F(t)$ can be attributed to the increase of capillarity force due to the decrease of pore sizes in a weakly vibrated medium, as well as to other mechanisms discussed in Refs. [44-48], but the origin of equality [Eq. (17)] is not clear. The lack of knowledge does not permit us to estimate the equilibrium height of the wetting front in a weakly vibrated medium, if it exists.

Although our experiments do not permit us to determine the creep $\mu$ directly, putting the scaling exponent [Eq. (8)] in Eq. (13) with $d=2$ and $n=2$, we obtain $\mu \approx 1$. This value, 
however, should be verified by direct measurements of the creep motion in the forced imbibition or drainage experiments.

\section{CONCLUSIONS}

Summarizing, we report the first observation of an almost steady motion of wetting front in a weakly vibrated granular medium. The front moves due to avalanches of localized intermittent advances directed at $45^{\circ}$ to the vertical axis. It should be emphasized that, while the data reported in Sec. III were obtained with cells of the same dimension filled with the same sand, the motion of wetting fronts with constant velocities was also observed in the preliminary experiments with cells of different apertures filled up to different sand packing densities. This suggests that the nonthermal creep regime we have studied here is quite widespread (and perhaps universal) in weakly vibrated granular systems. However, comprehensive studies of this phenomenon are needed to determine the conditions for its observation, as well as the dependence of creep parameters on the vibration features and the properties of granular media.
We suggest that the steady motion of a wetting front in a weakly vibrated granular medium can be treated as a nonthermal creep associated with density fluctuations in weakly vibrated granular media. In this context, the creep velocity is found to obey an analog of the Arrhenius law in which the compactivity of weakly vibrated granular media plays the role of effective temperature. Furthermore, our data suggest that the compactivity is a linear function of the gravitational acceleration, $X \propto G$, while the creep exponent is estimated to be $\mu \approx$ 1. We expect that these findings provide insights to the physics of granular media and nonthermal creep phenomena and will stimulate further theoretical and experimental research in these areas.

\section{ACKNOWLEDGMENTS}

This work was supported by the CSIC (España)CONACYT (México) under Project No. J101.390, the FONCICYT (México-European Union) under Project No. 96095, and the Government of Mexico City under Grant No. PICCT08-64.
[1] M. Alava, M. Dubé, and M. Rost, Adv. Phys. 53, 83 (2004).

[2] Th. Delker, D. B. Pengra, and P. Wong, Phys. Rev. Lett. 76, 2902 (1996).

[3] M. Dubé, M. Rost, and M. Alava, Eur. Phys. J. B 76, 691 (2000).

[4] Ch.-H. Lam and V. K. Horváth, Phys. Rev. Lett. 85, 1238 (2000); D. Geromichalos, F. Mugele, and St. Herminghaus, ibid. 89, 104503 (2002); S. Supple and N. Quirke, ibid. 90, 214501 (2003); Y. Mélean, D. Broseta, A. Hasmy, and R. Blossey, Europhys. Lett. 62, 505 (2003).

[5] Y. Méheust, G. Løvoll, K. J. Måløy, and J. Schmittbuhl, Phys. Rev. E 66, 051603 (2002).

[6] A. S. Balankin, A. Bravo-Ortega, and D. Morales, Philos. Mag. Lett. 80, 503 (2000); A. S. Balankin, O. Susarrey, and J. M. Gonzáles, Phys. Rev. Lett. 90, 096101 (2003); E. N. de Azevedo, L. R. Alme, M. Engelsberg, J. O. Fossum, and P. Dommersnes, Phys. Rev. E 78, 066317 (2008); A. M. Miranda, I. L. MenezesSobrinho, and M. S. Couto, Phys. Rev. Lett. 104, 086101 (2010).

[7] M. Dubé, S. Majaniemi, M. Rost, M. J. Alava, K. R. Elder, and T. Ala-Nissila, Phys. Rev. E 64, 051605 (2004).

[8] J. Soriano, A. Mercier, R. Planet, A. Hernández-Machado, M. A. Rodríguez, and J. Ortín, Phys. Rev. Lett. 95, 104501 (2005); S. Santucci, R. Planet, K. Jørgen Måløy, and J. Ortín, Europhys. Lett. 94, 46005 (2011).

[9] M. Sánchez, A. Medina, and A. Rojano, Rev. Mex. Fis. 51, 349 (2005).

[10] A. S. Balankin, R. Paredes, O. Susarrey, D. Morales, and F. Castrejon, Phys. Rev. Lett. 96, 056101 (2006).

[11] S. S. Le Guen and A. R. Kovscek, Transp. Porous Media 63, 127 (2006).

[12] M. Pradas, J. M. López, and A. Hernández-Machado, Phys. Rev. E 76, 010102R (2007).

[13] M. Pradas and A. Hernández-Machado, Phys. Rev. E 74, 041608 (2006); R. Planet, M. Pradas, A. Hernández-Machado, and
J. Ortín, ibid. 77, 056312 (2007); M. Pradas, A. HernándezMachado, and M. A. Rodríguez, ibid. 77, 056305 (2008).

[14] M. Pradas, J. M. López, and A. Hernández-Machado, Phys. Rev. E 80, 050101(R) (2009).

[15] J. M. López, M. Pradas, and A. Hernández-Machado, Phys. Rev. E 82, 031127 (2010).

[16] R. Planet, St. Santucci, and J. Ortín, Phys. Rev. Lett. 102, 094502 (2009); 105, 029402 (2010); J. Contam. Hydrol. 120-121, 157 (2011); G. Pruessner, Phys. Rev. Lett. 105, 029401 (2010).

[17] M. Dubé, B. Chabot, C. Daneault, and M. Alava, Pulp Pap. Can. 169, 24 (2005).

[18] M. Rost, L. Laurson, M. Dubé, and M. Alava, Phys. Rev. Lett. 98 , 054502 (2007); M. Dubé, C. Daneault, V. Vuorinen, M. Alava, and M. Rost, Eur. Phys. J. B 56, 15 (2007).

[19] Z. T. Karpyn and M. Piri, Phys. Rev. E 76, 016315 (2007); 76, 016316 (2007); B. Aminzadeh and D. A. DiCarlo, Vadose Zone J. 9, 588 (2010).

[20] C. Ulas Hatiboglu and T. Babadagli, Phys. Rev. E 77, 066311 (2008).

[21] C. Jian-Chao, Y. Bo-Mingand, M. Mao-Fei, and L. Liang, Chin. Phys. Lett. 27, 054701 (2010); J. Cai, B. Yu, M. Zou, and L. Luo, Energy Fuels 24, 1860 (2010); D. Ch. Standnes, ibid. 24, 2980 (2010); Chem. Eng. Sci. 65, 5178 (2010).

[22] S. Gruener and P. Huber, Phys. Rev. Lett. 103, 174501 (2009); S. Gruener, T. Hofmann, D. Wallacher, A. V. Kityk, and P. Huber, Phys. Rev. E 79, 067301 (2009); P. Gaillard, Y. Saito, and O. Pierre-Louis, Phys. Rev. Lett. 106, 195501 (2011).

[23] M. Lago and M. Araujo, Physica A 289, 1 (2001).

[24] N. Fries and M. Dreyer, J. Colloid Interface Sci. 338, 514 (2009).

[25] L. A. N. Amaral, A.-L. Barabási, S. V. Buldyrev, S. Havlin, and H. E. Stanley, Phys. Rev. Lett. 72, 641 (1994).

[26] A.-L. Barabási and H. E. Stanley, Fractal Concepts in Surface Growth (Cambridge University Press, Cambridge, 1995). 
[27] D. Bonn, J. Eggers, J. Indekeu, J. Meunier, and E. Rolley, Rev. Mod. Phys. 81, 739 (2009).

[28] L. A. N. Amaral, A.-L. Barabási, S. V. Buldyrev, S. T. Harrington, S. Havlin, R. Sadr-Lahijany, and H. E. Stanley, Phys. Rev. E 51, 4655 (1995).

[29] A. Dougherty and N. Carle, Phys. Rev. E 58, 2889 (1998).

[30] L. A. N. Amaral, A.-L. Barabási, H. A. Makse, and H. E. Stanley, Phys. Rev. E 52, 4087 (1995).

[31] P. Chauve, T. Giamarchi, and P. Le Doussal, Phys. Rev. B 62, 6241 (2000).

[32] A. B. Kolton, A. Rosso, and T. Giamarchi, Phys. Rev. Lett. 94, 047002 (2005)

[33] F. Caysol, D. Ravelosona, C. Chappert, J. Ferré, and J. P. Jamet, Phys. Rev. Lett. 92, 107202 (2004).

[34] A. Petković and T. Nattermann, Phys. Rev. Lett. 101, 267005 (2008).

[35] J. Kierfeld, H. Nordborg, and V. M. Vinokur, Phys. Rev. Lett. 85, 4948 (2000).

[36] A. B. Kolton, A. Rosso, T. Giamarchi, and W. Krauth, Phys. Rev. B 79, 184207 (2009).

[37] D. A. Gorokhov, D. S. Fisher, and G. Blatter, Phys. Rev. B 66, 214203 (2002).

[38] J. R. Thompson, J. G. Ossandon, L. Krusin-Elbaum, K. J. Song, D. K. Christen, and J. L. Ullmann, Appl. Phys. Lett. 74, 3699 (1999).

[39] M. Schröter, D. I. Goldman, and H. L. Swinney, Phys. Rev. E 71, 030301(R) (2005).

[40] E. R. Nowak, J. B. Knight, E. Ben-Naim, H. M. Jaeger, and S. R. Nagel, Phys. Rev. E 57, 1971 (1998).

[41] M. Nicodemi, A. Coniglio, and H. J. Herrmann, Phys. Rev. E 59, 6830 (1999).

[42] A. Janda, D. Maza, A. Garcimartín, E. Kolb, J. Lanuza, and E. Clément, Europhys. Lett. 87, 24002 (2009).

[43] A. Garcimartín, I. Zuriguel, L. A. Pugnaloni, and A. Janda, Phys. Rev. E 82, 031306 (2010).

[44] T. Hamida and T. Babadagli, in SPE Asia Pacific Oil and Gas Conference and Exhibition, 5-7 April 2005, Jakarta, Indonesia (Society of Petroleum Engineers, Allen, TX, 2005), 10.2118/92124-MS.

[45] T. Hamida and T. Babadagli, Transp. Porous Media 70, 231 (2007).

[46] T. Hamida and T. Babadagli, Colloids Surf., A 316, 176 (2008).

[47] T. Hamida and T. Babadagli, Ultrason. Sonochem. 15, 274 (2008).

[48] K. Naderi and T. Babadagli, Ultrason. Sonochem., 17, 500 (2010).

[49] H. L. Chen, L. R. Lucas, L. A. D. Nogaret, H. D. Yang, and D. E. Kenyon, SPE Reservoir Eval. Eng. 3, 16 (2001); H. Karimaie, O. Torsaeter, M. R. Esfahani, M. Dadashpour, and S. M. Hashemi, J. Pet. Sci. Eng. 52, 297 (2006); D. Ch. Standnes, Energy Fuels 24, 2980 (2010); R. Meher, M. N. Mehta, and S. K. Meher, Int. J. Appl. Math. Mech. 7, 29 (2011).

[50] M. M. Kohonena, D. Geromichalosb, M. Scheelb, C. Schierb, and S. Herminghaus, Physica A 339, 7 (2004);
Y. G. Tselishchev, V. A. Valtsifer, and O. G. Vasileva, Russ. J. Appl. Chem. 79, 1924 (2006); H. M. Scheel, R. Seemann, M. Brinkmann, M. D. Michiel, A. Sheppard, B. Breidenbach, and S. Herminghaus, Nat. Mater. 7, 189 (2008).

[51] H. Abou-Chakra, J. Baxter, and U. Tuzun, Adv. Powder Technol. 15, 63 (2004); M. Gan, N. Gopinathan, X. Jia, and R. A. Williams, KONA 22, 82 (2004).

[52] The overhangs were neglected.

[53] O. Chapuis and M. Prat, Phys. Rev. E 75, 046311 (2007).

[54] In some experiments, the highest parts of the moving wetting front achieve the top of the cell after $25 \mathrm{~min}$, such that the water escapes from the cell through the top gap.

[55] [http://www.trusoft-international.com] (BENOIT1.3, 2011); W. Seffens, Science 285, 1228 (1999).

[56] A. Arnéodo, E. Bacry, P. V. Graves, and J. F. Muzy, Phys. Rev. Lett. 743293 (1995); I. Simonsen, A. Hansen, and O. M. Nes, Phys. Rev. E 58, 2779 (1998); W. Lui, Int. Manage. Rev. 3, 33 (2001); A. Chamoli, Earth Sci. India 12, 258 (2008).

[57] J. J. Ramasco, J. M. López, and M. A. Rodríguez, Phys. Rev. Lett. 54, 2199 (2000).

[58] S. V. Buldyrev, A.-L. Barabási, S. Havlin, J. Kenész, H. E. Stanley, and H. S. Xenias, Physica A 191, 220 (1992).

[59] We noted that the process of grain rearrangement in a weakly vibrated medium is very slow (weeks or months) and so we assume that the equilibrium height of the wetting front cannot be achieved in our experiments due to the laboratory restrictions.

[60] R. C. Acharya, S. E. A. T. M. van der Zee, and A. Leijnse, Adv. Water Res. 27, 707 (2004); M. Gladkikh and S. Bryant, J. Colloid Interface Sci. 288, 526 (2005); Adv. Water Res. 30, 249 (2007); M. Gladkikh and S. L. Bryant, J. Pet. Sci. Eng. 52, 19 (2006); J. J. Milczarek, I. F. Kirejczyk, and J. Zoladek, Acta Phys. Polonica A 113, 1245 (2008).

[61] D. S. Fisher, Phys. Rev. Lett. 56, 1964 (1986).

[62] P. Philippe and D. Bideau, Europhys. Lett. 60, 677 (2002).

[63] P. Richard, M. Nicodemi, R. Delannay, P. Ribière, and D. Bideau, Nat. Mater. 4, 121 (2005).

[64] M. Mitarai and F. Nori, Adv. Phys. 55, 1 (2006).

[65] While for a specific system, a more appropriate control parameter could be $\Gamma=G \omega$ (J. A. Dijksman and M. van Hecke, Europhys. Lett. 88, 44001 2009); or the dimensionless energy $E$, injected in the granular system at lift-off (F. Ludewig, S. Dorbolo, T. Gilet, and N. Vandewalle, Europhys. Lett. 84, 44001, 2008), we have no matter to discuss the most appropriate control parameter associated with $T_{\text {eff }}$, because of the small range of gravitational acceleration explored in this work.

[66] S. F. Edwards and R. B. S. Oakeshott, Physica A 157, 1080 (1989); A. Mehta and S. F. Edwards, ibid. 157, 1091 (1989); S. F. Edwards and C. C. Mounfield, ibid. 210, 290 (1994); S. F. Edwards, J. Stat. Phys. 116, 29 (2004).

[67] A. Barrat, J. Kurchan, V. Loreto, and M. Sellitto, Phys. Rev. E 63, 051301 (2001); V. Colizza, A. Barrat, and V. Loreto, ibid. 65, 050301R (2002). 